\title{
Computer-Assisted Intuitive Breast Surgery Planning Using Three-Dimensional Surface Imaging
}

\author{
Laszlo KOVACS, Fee ARMBRECHT, Stefan RAITH, Alexander VOLF, Nikolaos A. PAPADOPULOS, \\ and Maximilian EDER \\ Research Group Computer Aided Plastic Surgery - CAPS, \\ Clinic for Plastic Surgery and Hand Surgery, Technical University Munich, Germany
}

\begin{abstract}
Introduction: Both patients and surgeons have a strong desire to anticipate the post-operative outcome before surgery. With the help of digital photography it is possible to "simulate" the surgical result by morphing two-dimensional images with adequate software applications. The introduction of three-dimensional (3-D) surface imaging offers a feasibility of virtual deformation with haptic devices and specific software. The aim of this study was to investigate the potential clinical application of preoperative surgical modelling regarding relative advantages, disadvantages, reproducibility (precision), accuracy and limitations of the method.
\end{abstract}

Materials and Methods: We would like to present a standardized protocol for virtual 3-D surgical planning by modelling the aspired postoperative using a force feedback system (Fig. 1). Result of five breast augmentation (Fig. 2) and five breast reductions (Fig. 3) and compared it with the real surgical result. The postoperative result of breast augmentation and breast reduction patients are virtually planned with the new 3-D technology and the expressiveness of the method are evaluated. The differences in breast volume, linear distances and the 3-D shape of the breast are compared to the real surgical outcome.

Results: Patients are impressed by the 3-D features: rotation of images, viewing from different angles, deformability of the body contour. The 3-D technology supplies the surgeon with accurate assessment of surface, volume, shape, distances and an acceptable reproducibility. Only the 3-D shape analysis showed differences in the clinical application study. The introduction of 3-D surface imaging to medical applications in the last few years offers a feasibility of virtual deformation with haptic interfaces and specific software which was up to now only designed for industrial, technical, mathematical and graphical applications. The combination of data processing using haptic interfaces, compact application packages and the potential visualisation of the aspired result enables the surgeon a preoperative surgical planning.

Conclusion: 3-D intuitive breast surgery planning is sufficiently precise and accurate and both, patients and surgeon can benefit from this new 3-D technology. But the physical attributes of the human tissue are not taken into account in the preoperative planning, thus the expression "surgery simulation" cannot be used. We prefer the term of "Intuitive 3-D Surgery Planning" because the subjective imagination of the surgeon still defines the postoperative result and further applications has to take place in the future.

Keywords: breast, 3-D surface imaging, breast surgery, force feedback, surgical planning

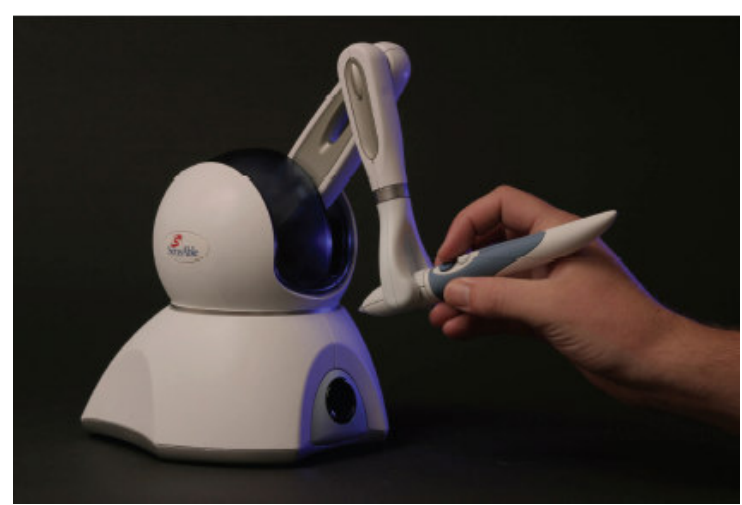

Fig. 1: The used force feedback system Phantom® Omni TM and FreeForm $\circledast$ Concept ${ }^{T M}$, SensAble Technologies, USA 

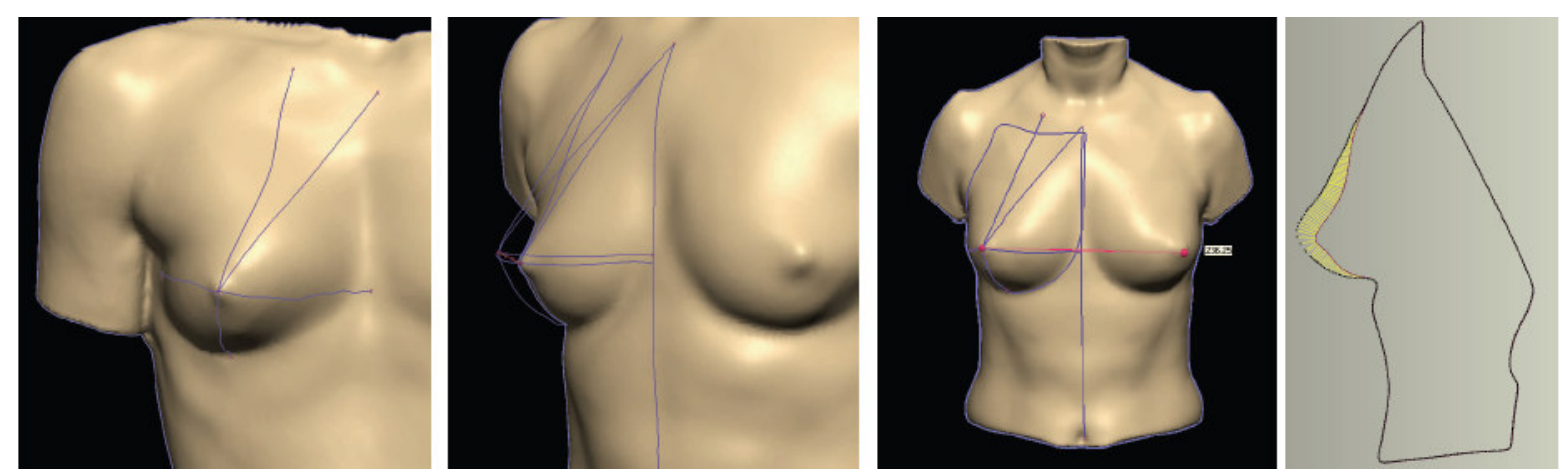

Fig. 2 3-D virtual planning of breast augmentation surgery
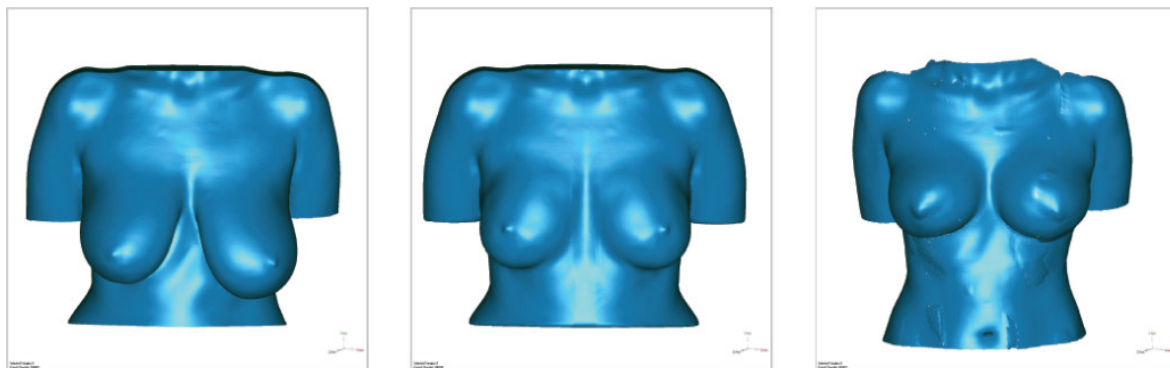

Fig. 3 3-D virtual planning of breast reduction surgery

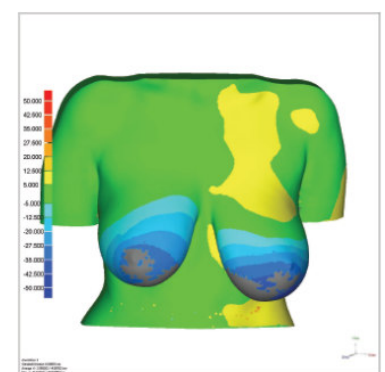

